\title{
Centros comerciales y dinámica urbana en el contexto metropolitano español. Estudio de casos
}

\author{
Shopping centres and urban dynamics in the Spanish metropolitan \\ context. Case study
}

\author{
Amalia Vahí Serrano ${ }^{1}$ \\ Claudia Hurtado Rodríguez $z^{2}$ (D)
}

\section{Resumen}

La mayor parte de la población en España reside y trabaja en las ciudades aunque la información que se desprende de la movilidad cotidiana denota que los mercados de vivienda y trabajo trasvasan la escala municipal. Este hecho es indicativo de dinámicas interurbanas compartidas que a su vez promueven flujos de desplazamientos y una demanda sin precedentes de infraestructuras y equipamientos. Junto a la identificación de la realidad supraurbana formal y funcionalmente unitaria que denominamos metropolitana, urge profundizar en los comportamientos de diferentes protagonistas que contribuyen a la complejidad de estos ámbitos. Entre los grandes centros de actividad que potencian y dinamizan la movilidad en la escala metropolitana, los grandes equipamientos comerciales cobran un fuerte protagonismo sobre la ciudad metropolitana española del siglo XXI. El presente texto se hace eco de ello ${ }^{3}$ y explora la interacción de estos con los nuevos escenarios de aglomeración, que se pueden llegar a ver condicionados o afectados en las estructuras y formas urbanas por la implantación de estos centros (en tanto que motores/ potenciadores de nuevos desarrollos de urbanización). La propuesta y los resultados invitan a la reflexión en el conjunto de un proyecto de largo recorrido en torno al modelo de ciudad sostenible sobre el que se trabaja desde diferentes disciplinas e instancias de responsabilidad institucional.

Palabras clave: centros comerciales; procesos metropolitanos; infraestructuras; movilidad; articulación urbana.

\begin{abstract}
Most of the population in Spain lives and works in cities. However, the data regarding daily-life mobility shows features of the housing and labour markets that go beyond the municipal level. This is an indicator of shared inter-city dynamics that promote displacement while an unprecedented demand on infrastructure and facilities is created. Metropolitan areas are supra-urban realities, which are homogeneous from a formal and functional point of view and need to be identified. Moreover, an in-depth analysis is required to study the attitudes of the different figures that contribute to the complexity of these areas. There are various hubs of activity that foster and boost mobility on a metropolitan scale. These include retail parks that have become prominent in the context of metropolitan cities in the 21st Century Spain.

1 Departamento de Geografía, Historia y Filosofía. Universidad Pablo de Olavide, España. avahser@upo.es

2 Programa Medio ambiente y sociedad. Universidad Pablo de Olavide, España. churrod@upo.es

3 El presente texto resulta de los avances de la investigación en el marco del Proyecto "Dinámicas y transformaciones territoriales, funcionales y sociales de las áreas metropolitanas españolas en un horizonte de sostenibilidad", financiado en el marco del Plan Estatal de Investigación Científica y Técnica y de Innovación 2013-2016, código: CSO2014-55780-C3-1-P. que ha formado parte de un Proyecto Coordinado (IP Dr. José María Feria Toribio).
\end{abstract}


The purpose of this article is to discuss this reality, and to analyse the interaction between retail parks and potential urban agglomerations. The latter can be affected by the creation of retail parks, which promote new urban developments. The thought-provoking proposal and outcome presented in this article are also studied in the context of a long-term project that involves diverse disciplines and institutions associated with a sustainable city model.

Keywords: Shopping centres; metropolitan processes; infrastructures; mobility; urban articulation.

\section{Introducción}

Históricamente el urbanismo ha evolucionado de modo diverso a tenor de factores socioeconómicos y culturales, pero la tendencia generalizada a la concentración de hábitat y servicios permitió desde el siglo pasado modelizar y crear teorías explicativas respecto a la complejidad y diversidad del fenómeno (Dematteis, 1998; 2004; Hall, 2004). En lo que respecta a los grandes equipamientos aparecidos en la escena urbana y metropolitana, y las infraestructuras en que estos se apoyan, nutren y contribuyen al crecimiento de las ciudades deviniendo en transformaciones de la trama y la forma urbana, sujeta a una actividad inmobiliaria de intensidad variable. En relación a la percepción cortoplacista y falaz de la bonanza financiera (generalizada en el llamado mundo desarrollado) a finales de siglo XX y -muy claramente- en la década siguiente, los suelos se convirtieron en España -como en otros países- en recurso al alcance de las municipalidades y un jugoso asunto para las entidades financieras que lo adoptaron como principal valor de cambio (Tamames, 2009). De ese modo, el planeamiento urbanístico y la gestión municipal se posicionó con frecuencia en la vía del crecimiento y la expansión, carente de un modelo riguroso sobre la ciudad deseada. Con dichas ausencias y mediando solo en foros reducidos el pulso entre ciudad moderna y paradigma de la postmodernidad, a menudo se minusvaloró desde instancias responsables la incorporación del equilibrio ambiental (Burriel de Orueta, 2008), que debemos considerar necesario entre forma y función urbana. Todo ello ha dibujado y acentuado la desmesura de ciudades recrecidas y un déficit en el balance de las dinámicas derivadas de los flujos a esa escala. El equilibrio territorial en estos ámbitos -por tanto- se encuentra en entredicho toda vez que acusan disfunciones sociales, ambientales y económicas. La certeza sobre la dinámica desencadenada, los procesos de crecimiento descritos y el rol desempeñado por los grandes equipamientos se aprecia en los resultados y conclusiones de recientes investigaciones (Williams, Jenks \& Burton, 2001; Vahí y Feria, 2007; García Palomares y Gutiérrez Puebla, 2007).

La componente productiva, cimentada históricamente en estos escenarios urbanos metropolitanos sobre la industria, ha ido abriendo posibilidades de expansión en consonancia con los nuevos modelos productivos sucedidos desde finales del siglo XX. La expansión de los servicios desde mediados de los años 70 del pasado siglo ha promovido en nuestra opinión las mayores transformaciones urbanas en las últimas décadas, y durante algún tiempo ha protagonizado una suerte de matrimonio conveniente con los agentes urbanísticos (administraciones con competencias) que basaron el progreso y el desarrollo en estrategias de capitalización y fomento del desarrollo de nuevos suelos (a partir del planeamiento). La implicación de los municipios en torno a las grandes ciudades ha contribuido de modo imparable a estas grandes transformaciones con una redistribución funcional de las actividades productivas y servicios en una suerte de contraurbanización, de acuerdo con algunos autores (Arroyo, 2001). Al margen del debate epistemológico sobre estas cuestiones interpretativas sobre la dinámica urbana y urbanizadora, lo cierto es que ésta ha devenido en las últimas décadas del siglo XX en oportunidades tanto para actores de las administraciones como desde el ámbito privado, que han activado cambios de uso y creación de suelos fuera del plano urbano para servicios demandantes de grandes parcelas y garantías de accesibilidad/conectividad. Dicha dinámica ha desembocado en la aparición de nodos que en ocasiones trascienden topológicamente la determinación de los lugares puntuales para convertirse en sucesión de localizaciones de empresas, equipamientos, dotaciones -en definitiva- que acaban por conformar y ser reconocidos como parques (comerciales, tecnológicos, empresariales...), distritos (industriales, de negocios, financieros,...) y otras acepciones evocadoras del carácter extensivo e intensivo de los emplazamientos (Paris, 2013). Sin menoscabo de otras tipologías contempladas en el marco de la investigación (complejos hospitalarios y universitarios o parques tecnológicos, industriales y logísticos), el presente artículo se centra en los grandes equipamientos comerciales que se hallan sin excepción en todas las áreas identificadas en el proyecto referido en el apartado de reconocimientos (CSO2014-55780-C3-1-P). Desde los campos de la Geografía y el Urbanismo se trabaja en la proyección ambiental de los centros comerciales en tanto que potenciales motores de desarrollos urbanísticos existentes o de nueva creación (Vahí y Feria, 2007). Son 
grandes protagonistas del actual modelo imperante en los espacios de mercado tal y como apuntan las cifras de la asociación empresarial del ramo que refuerza lo que ya evidencian el planeamiento municipal de muchas ciudades españolas y la propia recopilación y tratamiento de datos que nuestro proyecto incluye. La Asociación Española de Centros Comerciales (AECC) pone de relieve los más de quince millones de metros cuadrados de centros comerciales en nuestro país a mediados de 2015 , lo que triplica la superficie ocupada por estos centros a comienzos de los años 80 . El número de establecimientos pasó de 20 a $546^{4}$ debiéndose tener en cuenta que esta fórmula comercial se ha prodigado en estos más de 35 años en todas las ciudades grandes y medianas del país, la mayor parte en los contextos metropolitanos identificados (Figura 1). La cifra actual es similar aun con ajustes oportunos para el presente análisis, como el que se hayan agrupado algunos establecimientos que comparten emplazamiento, dentro de un sector urbano, parque comercial,... (Vahí, Rodríguez y Hurtado, 2015).

Más allá de las dimensiones y la superficie que ocupa este segmento de los servicios, observamos su incidencia sobre la demanda de sistemas generales a la escala local y metropolitana, cuyos costes económicos quedan desproporcionada y desigualmente distribuidos entre las administraciones y los promotores. No es objeto de este artículo bucear en aspectos propios de aplicación de la disciplina urbanística sensu estricto, pero sí incidir en aspectos que repercuten y se alejan de un modelo urbano desarrollado, socialmente cohesionado y ambientalmente sostenible.

Figura 1. Evolución de los Centros Comerciales en Áreas Metropolitanas Españolas

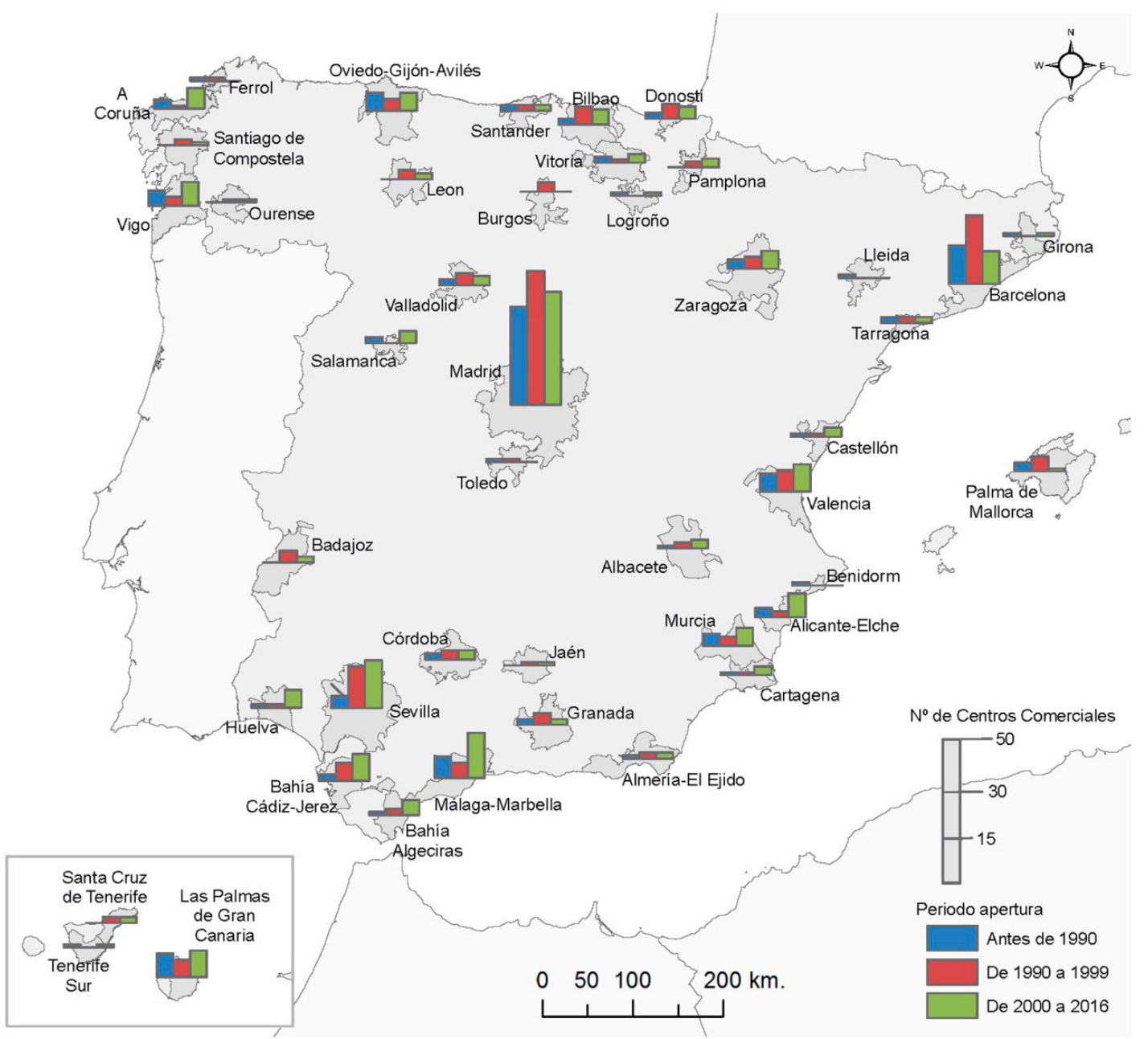

Fuente: Directorio de AECC 2012, webs comerciales (2016). Elaboración propia.

\footnotetext{
4 Dada la diversidad de criterios empleados sobre la definición y superficie se ha optado convencionalmente por uniformar nuestro análisis contemplando los CC a partir de $5000 \mathrm{~m}^{2}$ de SBA.
} 
Estos centros de actividad comercial (parques empresariales y de servicios, complejos comerciales y de ocio) motivan un importante número visitas, como evidencia el Observatorio de la Movilidad Metropolitana $[\mathrm{OMM}]$ con la difusión de informes al respecto (OMM del Gobierno de España, desde 2002) o los estudios de mercados y encuestas de instancias diversas (Informes de AECC, encuestas de asociaciones de consumidores, etc.). Siendo una realidad intensa y extendida, aun con el paso de los años y la constatación de efectos no deseados se mantiene la constante, casi en el 100\% de los casos, de desplazamientos motorizados en vehículos privados. La movilidad urbana cotidiana participa en una suerte de bucle según el cual el consumo de nuevos suelos urbanos y urbanizables suscita la dotación de infraestructuras y servicios adecuados a los usos presentes y futuros, y -con frecuencia- atraen sucesivamente y en tiempo variable nuevas inversiones, nuevos usos residenciales y terciarios. Cabe hablar de crecimiento, más que de desarrollo urbano, y en cualquier caso este bucle se retroalimenta merced a la oferta inagotable de infraestructuras del transporte, creadas ex novo o por ampliación de las existentes. Tal circunstancia ya se aprecia en el comienzo de siglo, en que algunos investigadores (como Serrano, Gutiérrez y otros) aprecian en nuestro país un inusitado desarrollo de vías de alta capacidad (autovías, autopistas y desdobles de viario perimetral urbano) en la práctica totalidad del viario con intensidad media superior a 8000 vehículos diarios, buena parte de las cuales se incluyen las áreas metropolitanas del país, razón por la que la temporalidad de los desplazamientos más abundantes se ha reducido considerablemente (Serrano, 2001). Sin embargo, también ha quedado constatada la dependencia del mercado residencial (sujeto a modelos más o menos difusos) que intensifican la dependencia del automóvil privado (Serrano, 2006), lo que -a la postre- ha multiplicado la gravedad del problema: al descuido por la intermodalidad del transporte y el consiguiente celo por garantizar la infraestructura del automóvil, se une el estímulo de pautas de consumo de tiempo y espacio, para los que productos inmobiliarios (residencial, comercio, empresarial, ocio..) están disponibles. El PEIT (Plan Estratégico de Infraestructuras y Transporte, 2004) de alcance estatal se propuso a fin de atender las carencias y minimizar sus efectos, aunque el progreso es ciertamente lento tal y como puede percibirse hoy.

El modelo de crecimiento urbano imperante en la actualidad es consecuencia de la falta de respuestas a las alternativas para contrarrestar el crecimiento expansivo. Cuestiones sociológicas y ambientales de diverso tipo convergen en aspectos relacionados con la presencia de los grandes equipamientos comerciales; todas incitan a reconducir el consumo de tiempo y espacio toda vez que la ciudad arroja señales para declarar su incapacidad de gestión en cuestiones como la libertad de establecimiento, la garantía de accesibilidad o la dotación infraestructural para dar cobertura a estas centralidades. La ciudad metropolitana, en tanto que espacio de trabajo e intercambios de bienes, información y personas, y lugar de residencia, suscita graves problemas por las distancias a salvar y las tensiones derivadas del uso creciente del automóvil privado frente a una intermodalidad insuficientemente extendida. En este sentido, el objetivo descrito no renuncia a la perspectiva de criterios de equidad y cohesión social que propugnan un modelo urbano sostenible del que los grandes centros de actividad no deberían escapar por muchos condicionantes o contradicciones que se les presenten (Guy, 2006). El proyecto del que parte el presente artículo incluye los grandes centros de actividad al conformar uno de los pilares sobre los que actualmente están pivotando procesos de crecimiento y expansión urbana, algo que les dota de peso en las tomas de decisiones acerca del modelo de ciudad. Si bien el proyecto incluye otras tipologías, los grandes equipamientos comerciales son posiblemente los más significativos dada la variedad tipológica y la afluencia masiva que diariamente generan hacia ellos (personas, mercancías, servicios).

\section{Metodología}

El artículo se propone mostrar el papel actual de los grandes centros comerciales minoristas sobre el crecimiento urbano y sus repercusiones sobre el trazado de la ciudad, en particular las infraestructuras y nuevos servicios asociados a los (hasta ahora) ineludibles desplazamientos motorizados. Abundar en estas cuestiones nos permite plantear un objetivo más, que es el de reforzar la reflexión sobre la actual ciudad metropolitana en tanto que retroalimenta pautas de crecimiento (con el consiguiente consumo de suelos y recursos) que se aleja del principio de sostenibilidad ambiental y de un modelo territorial socialmente cohesionado.

La metodología diseñada para ahondar en estas cuestiones y poner de relieve la simbiosis descrita, incorpora el manejo de estadísticas elaboradas y en permanente revisión lo que facilita y contrasta con el análisis espacial. En primer lugar planteamos que nuestro punto de partida para la delimitación de las 
áreas metropolitanas es el que se viene siguiendo en el conjunto del proyecto en que se insertan estos resultados. La delimitación de las áreas metropolitanas españolas adoptada en este caso fue introducida años atrás en la Geografía de nuestro país por diversos autores (Feria Toribio, 2004; Salom y Albertos, $2014, \ldots$ ) y se inspira en el modelo contrastado y probado décadas atrás de las unidades territoriales administrativas en Estados Unidos (Berry, Goohen \& Goldstein, 1970). Aun cuando en el momento actual los expertos norteamericanos revisan los factores que intervienen en dicha delimitación, los criterios de la práctica estadística de las ciudades norteamericanas de indiscutible solidez da pie a la extrapolación del método a la línea seguida en el proyecto en que se encuadra este artículo. La traslación de dicha metodología y un profundo conocimiento sobre la delimitación de las áreas españolas se encuentra en la base del presente artículo que se apoya igualmente en la interacción de los mercados de vivienda y trabajo así como los flujos desencadenados a consecuencia de la movilidad cotidiana (Feria Toribio, 2008). Partiendo de la dinámica y complejidad de los espacios de centralidad como son las grandes ciudades españolas, la delimitación tomada para nuestros ámbitos de estudio responde a la aplicación de un algoritmo que expresa umbrales dentro de los cuales se identifican ámbitos municipales con mercados de residencia y trabajo compartidos, hasta el punto de generar pautas relevantes de movilidad cotidiana ${ }^{5}$. El resultado diferencia potentes focos urbanizados atendidos por servicios y centros de producción que contienen a más del 70 \% de la población española. Esos focos, a su vez, guardan diferencias en virtud no sólo del tamaño y diversidad funcional sino también en función de otros rasgos de carácter formal que contribuyen a reconocerlos aún más en su singularidad. Así, en España distinguimos regiones metropolitanas (Madrid y Barcelona), a diferencia de áreas que contienen al tiempo una diversidad reseñable: áreas metropolitanas consolidadas, centralizadas, policéntricas, reticulares, incipientes (Feria Toribio, 2008).

En cuanto al planteamiento metodológico, hay que destacar que este trabajo parte del tratamiento y explotación de una potente base de datos espacial desarrollada en anteriores investigaciones a partir de la consulta exhaustiva de diversos directorios oficiales (Vahí, Rodríguez y Hurtado, 2015). Gracias a este trabajo previo se ha podido identificar, caracterizar y localizar los grandes centros de actividad en el marco de las áreas metropolitanas españolas, tareas para las cuales han sido determinantes las fuentes ministeriales no solo para el aparato cartográfico (que se comenta más adelante) sino también en el aporte de catálogo de centros sanitarios (Ministerio de Sanidad, Servicios Sociales e Igualdad [MSSSI]). Igualmente ciertas entidades sectoriales han sido consultadas por imprescindibles: Asociación de Centros de Transporte de España, Asociación de Parques Científicos y Tecnológicos de España (APTE) y Fundación UNIVERSIA.

De forma paralela, y en cuanto a la dimensión física del fenómeno metropolitano, se entiende el espacio urbano desde su naturaleza expansiva de forma horizontal a lo largo del territorio, aproximándonos a la definición de "mancha urbana", como la superficie ocupada como el espacio construido. En esta línea se decide acotar estos espacios gracias al uso del modelo de datos de ocupación del suelo CORINE Land Cover, (CLC) (Ministerio de Fomento-Instituto Geográfico Nacional (2016)). Sobre esta fuente espacial cabe puntualizar que aun presentando ciertos limitantes para estudios que requieren, por su aproximación o por necesidades de escala, de un alto grado de resolución espacial, se presenta en este caso como una fuente óptima por la posibilidad de distinguir, dentro de su nomenclatura jerárquica, los distintos tipos de tejido urbano, pero sobre todo porque se presenta como una fuente homogeneizada para todo el territorio europeo y español, algo que facilita estudio comparativo de los espacios metropolitanos abordados en este artículo. Por otro lado, el hecho de que este trabajo pretende relacionar las transformaciones sufridas por las macroformas urbanas con el desarrollo de los grandes centros de actividad a lo largo del territorio metropolitano hace que el análisis espacial planteado no requiera de una fuente con una escala más allá de la presentada por CLC (1:100.000).

En virtud de lo expuesto, el método propuesto presenta una doble aproximación acerca de la cuestión planteada. La primera parte pone en relieve la relación de los distintos componentes territoriales que actúan como ejes del desarrollo metropolitano mediante el análisis visual de la configuración de los mismos a lo largo del espacio metropolitano. En esta ocasión se tienen en cuenta la red principal de carretera, el tejido urbano, independientemente de la funcionalidad o naturaleza del mismo (residencial, industrial, comercial, etc.), y los distintos centros funcionales de actividad, conscientes del papel que cada uno de

\footnotetext{
5 Resultante de la consideración de una ciudad central superior a los 50.000 habitantes y las estadísticas municipales en relación a aquellos casos que guardan una contigüidad espacial y cumplen que: al menos un 15\% de la población ocupada trabaje en el municipio central o, en sentido contrario, que un $25 \%$ de la población que trabaja en el municipio, proceda del central. La cifra del $15 \%$ está en revisión pero hoy por hoy nos apoyaremos en ella.
} 
ellos tiene en la articulación del territorio, aunque en este caso se enfatiza especialmente la figura de los grandes centros comerciales. Esta fase del análisis se centra en las cuatro principales áreas metropolitanas españolas; Madrid, Barcelona, Valencia y Sevilla, tanto por la dimensión de las mismas como por formar los principales espacios donde se han consolidado los grandes centros funcionales a nivel nacional.

La segunda parte del análisis ofrece una visión más pormenorizada sobre la posible relación espacial entre los centros comerciales y dos componentes o ejes de desarrollo básicos para entender los procesos de expansión urbana en espacios metropolitanos; los grandes ejes de comunicación y la mancha o núcleo urbano principal. En relación a este último, hay que puntualizar que dentro de la diversidad que presentan las áreas metropolitanas españolas en relación a su organización espacial, existen casos en los que el grado de policentrismo es evidente. Es en estos espacios donde se ha realizado el análisis tomando para ello varios núcleos principales (Bahía de Cádiz-Jerez, Almería-El Ejido, Málaga-Marbella, Elche-Alicante, Oviedo-Gijón-Avilés, Vigo-Pontevedra).

En cuanto al criterio adoptado en el análisis, se decide tomar la proximidad espacial entre elementos con el limitante de tránsito a través de la red de carreteras. Esto se realiza sobre una base espacial en formato raster que consiste en sistema continuo de rejillas de 100x100 metros. La decisión de introducir en este modelo la red de carreteras para limitar la movilidad tiene su lógica el fuerte vínculo existente entre el uso del vehículo privado y el acceso a los grandes centros comerciales, y aunque dota de cierta complejidad al diseño del modelo, creemos necesario introducir este elemento frente al planteamiento convencional de distancia euclídea, o en línea recta en vistas a la probable distorsión que esta aproximación podría producir en los resultados (Figura 2)

Figura 2. Diferencia entre análisis de distancia euclídea (izquierda) y análisis a través de la red de carreteras (derecha), núcleo central del área metropolitana de Madrid
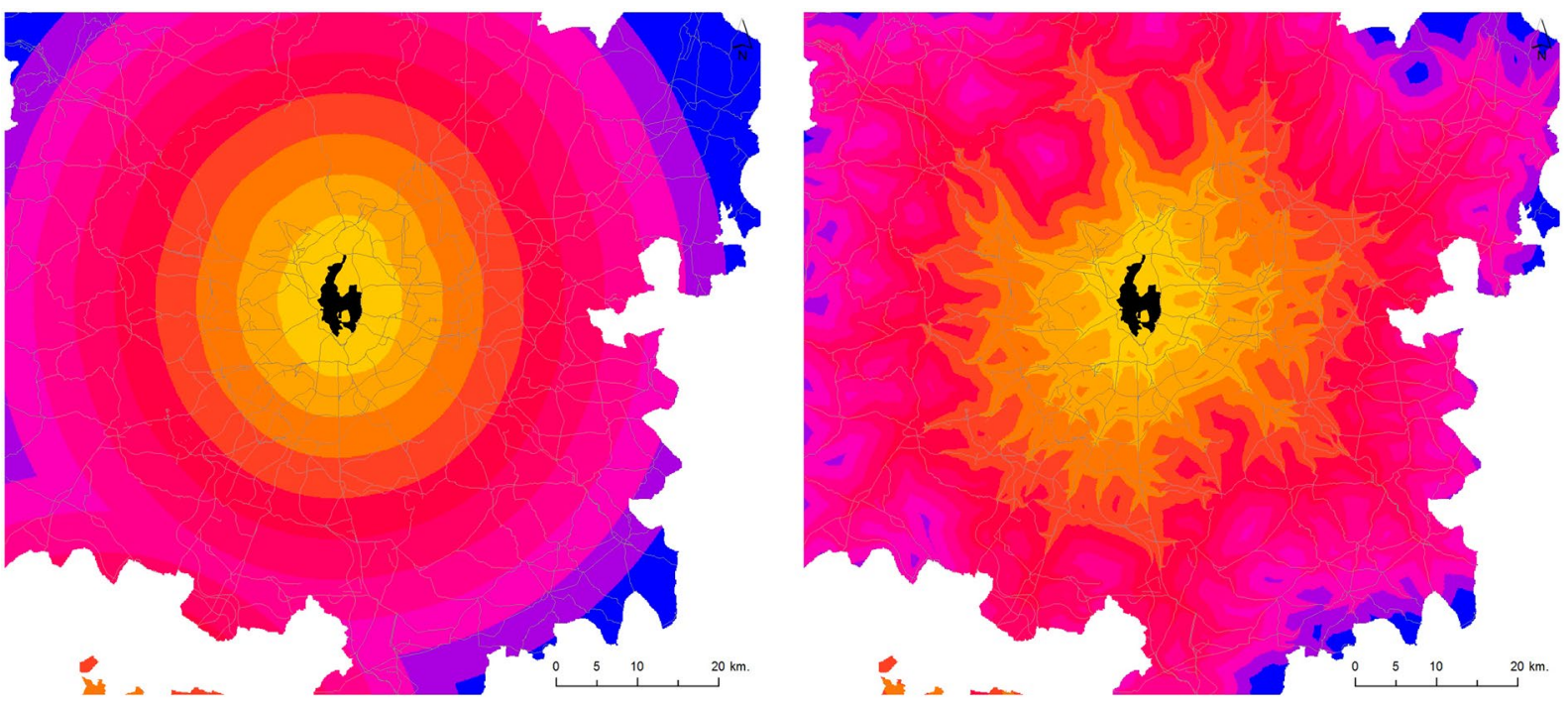

Fuente: CLC (Ministerio de Fomento-Instituto Geográfico Nacional (2016)

y BTN100 Ministerio de Fomento-Instituto Geográfico Nacional (2015). Elaboración propia.

Por otro lado, y en vistas a los buenos resultados obtenidos siguiendo este proceso, la posibilidad de realizar un análisis de proximidad a través de un modelo vectorial se planteaba como una tarea difícilmente abordable y poco recomendable en este caso teniendo en cuenta la dimensión de los espacios propuestos (un total de 44 áreas metropolitanas), fundamentalmente porque esta opción requiere de la preparación de una red de carreteras con continuidad topológica en todos los tramos de la misma.

De este modo, el desarrollo del proceso metodológico se plantea utilizando como base la red de carreteras de la Base Topográfica Nacional 1:100.000, (BTN100) (Ministerio de Fomento-Instituto Geográfico Nacional (2015). y las herramientas de análisis espacial de distancia "cost distance" del software ArcGIS 10.2, que permiten el cálculo de la distancia acumulada entre celdas en base a una superficie de coste. En este caso el coste menor es asignado para las autopistas y autovías, seguidas por las secundarias y por último un coste mucho mayor para el resto del territorio. 
En cuanto a los dos componentes territoriales que se han considerado en el análisis espacial, hay que señalar que, por un lado, el núcleo urbano principal o central se ha definido considerando como la principal mancha urbana o núcleo neurálgico de cada área metropolitana, delimitada a partir de la superficie urbana continua, según nomenclatura de CLC, para el año 2006. Teniendo en cuenta en este punto la consideración especial de los espacios metropolitanos policéntricos. Por otro lado, los principales ejes de comunicación se han establecido a partir de la extracción de autovías y autopistas de la red de carreteras de la BTN100.

Finalmente, hay que destacar el carácter espacio-temporal y dimensional de esta aproximación, ya que la base de datos diseñada nos proporciona información sobre el año de apertura y sobre la superficie de los centros comerciales, algo que sin duda va a enriquecer los resultados obtenidos ofreciendo una visión clave para entender la evolución de estos grandes centros a lo largo del proceso de metropolitanización.

\section{Resultados}

El papel de las infraestructuras del transporte y comunicaciones como potenciadoras de las dinámicas metropolitanas está sobradamente contrastado. Aun cuando las grandes ciudades actualmente acusan la persistencia de factores urbanísticos históricos con los que tratan de no romper (núcleo principal, central, emblemático y simbólico que permanece), la creciente dependencia de los desplazamientos en la escala metropolitana evidencia el protagonismo de dichos sistemas, que se incorporan, se adaptan e incluso abren posibilidades a nuevos conceptos e interpretaciones sobre la ciudad más allá del núcleo.

Muestras del papel trascendental de estos sistemas los proporcionan los grandes ejes del viario intraurbano, sobre todo en las ciudades más dinámicas, que acaban por extenderse más allá de los ensanches hacia nuevas periferias urbanas contribuyendo a la construcción metropolitana; o la proyección de nuevos corredores y circunvalaciones en torno a bolsas de suelo previstas para nuevos desarrollos. En ese escenario los grandes centros de actividad productiva (parques y polígonos industriales, logísticos) y de servicios (grandes centros comerciales, complejos hospitalarios, tecnológicos, investigación, universidades) han contribuido a la conformación de nuevas realidades urbanas, impulsando o refrenando procesos de crecimiento a través de vectores que se extienden intencionadamente entre las diferentes piezas del conjunto metropolitano. Así lo ponen de manifiesto los mapas de las áreas seleccionadas para la exposición de nuestros resultados (Figuras 8 a 11)

Poniendo el foco sobre los centros comerciales obtenemos los siguientes resultados para el conjunto de las 44 áreas metropolitanas españolas, a partir de un análisis de proximidad. Seleccionados dos criterios (proximidad a la vía de comunicación de primer nivel y proximidad al núcleo principal del área metropolitana), se han cruzado los datos con la cronología de apertura del establecimiento (Figuras 3-6). De resultas, se detecta un comportamiento constante y muy generalizado (salvo el caso de los grandes almacenes, como es sabido) en cuanto al emplazamiento periférico o sobre el territorio metropolitano, siempre con el respaldo de la corta distancia al viario principal.

Figura 3. Análisis de proximidad "centros comerciales-ciudad central", según fase de apertura

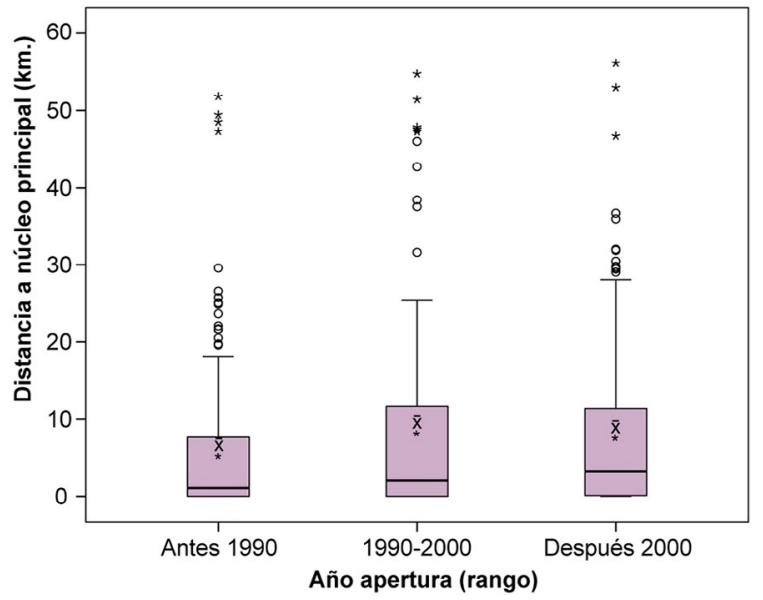

Fuente: CLC (Ministerio de Fomento-Instituto Geográfico Nacional (2016) y BTN100 Ministerio de Fomento-Instituto Geográfico Nacional (2015). AECC, 2012 y webs propias de enseñas centros comerciales. Elaboración propia. 
Sobre un viario de primer nivel se apoyaron los primeros grandes centros, facilitando la afluencia ante la atracción ejercida por los mismos. Sucesivas estrategias del sector comercial e inmobiliario a lo largo de los últimos cuarenta años han promovido aperturas en las proximidades de los centros pioneros, lo que a menudo ha inducido nuevos proyectos de infraestructuras de transporte rodado, retroalimentando así los procesos urbanísticos que trascienden a la ciudad para abrazar un ámbito metropolitano.

Figura 4. Análisis de proximidad "centros comerciales-viario de primer nivel" según fase de apertura

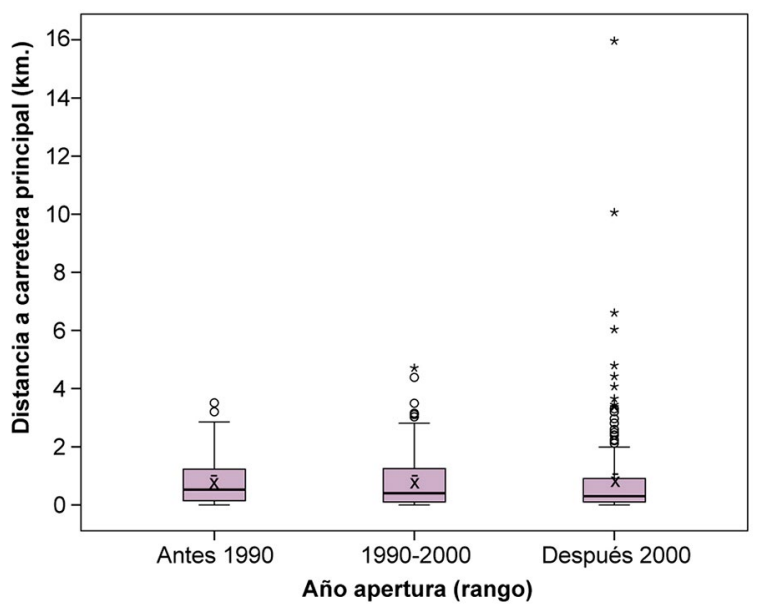

Fuente: CLC (Ministerio de Fomento-Instituto Geográfico Nacional (2016) y BTN100 Ministerio de Fomento-Instituto Geográfico Nacional (2015). AECC, 2012 y webs propias de enseñas centros comerciales. Elaboración propia.

Cuando se ha incluido en el análisis la dimensión de los centros comerciales (superficie bruta alquilable $)^{6}$ resulta una cierta coincidencia en las tendencias de localización respecto a los elementos que organizan el territorio metropolitano, de tal modo que los mayores establecimientos se encuentran a menor distancia de carreteras de primer orden que los restantes centros (Figura 6). Las dimensiones superiores están asociadas a fórmulas mixtas que aglutinan enseñas y tiendas franquiciadas, cadenas, sucursales, $\ldots$ que constituyen un referente en el campo de la confección, alimentación, ocio, etc. Esa diversidad es en sí misma una estrategia de promotores y comercializadores, que se aseguran elevadas afluencias (Figura 5, Figura 6).

Figura 5. Análisis de proximidad "centros comerciales-ciudad central" según tamaño del centro

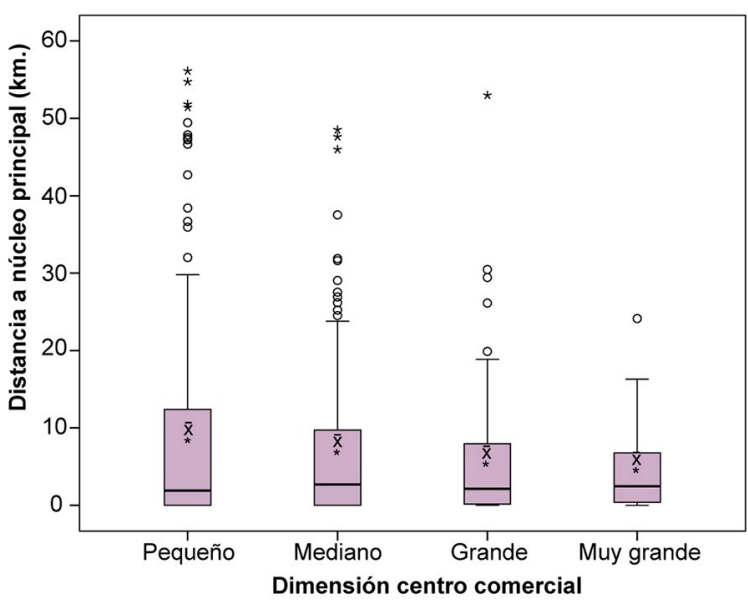

Fuente: CLC (Ministerio de Fomento-Instituto Geográfico Nacional (2016) y BTN100 Ministerio de Fomento-Instituto Geográfico Nacional (2015). AECC, 2012 y webs propias de enseñas centros comerciales. Elaboración propia.

6 Acuerdos alcanzados por International Council of Shopping Centers y las asociaciones de centros comerciales de países europeos; Pequeño de 5.000 a $19.999 \mathrm{~m}^{2}$, Mediano de 20.000 a $39.999 \mathrm{~m}^{2}$, Grande de 40.000 a $79.999 \mathrm{~m}^{2}$ y Muy Grande más de $80.000 \mathrm{~m}^{2}$. 
Figura 6. Análisis de proximidad de los CC a la vía de primer nivel según tamaño del centro

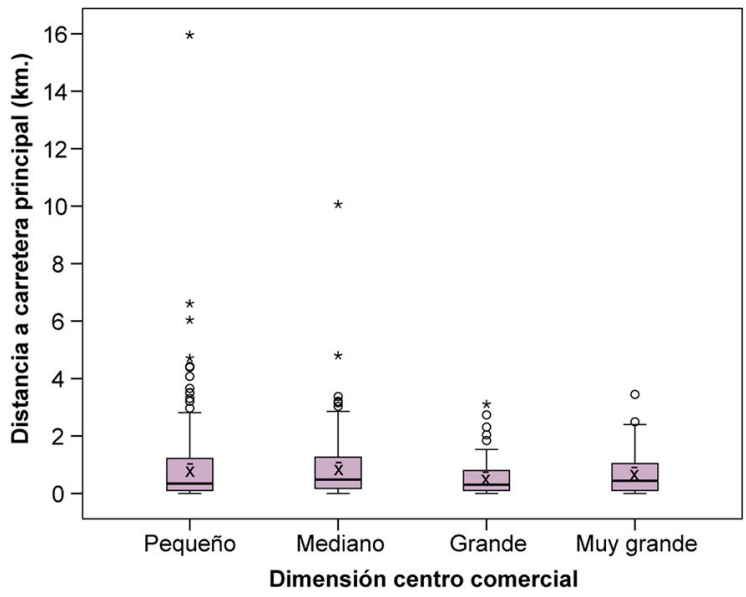

Fuente: CLC (Ministerio de Fomento-Instituto Geográfico Nacional (2016) y BTN100 Ministerio de Fomento-Instituto Geográfico Nacional (2015). AECC, 2012 y webs propias de enseñas centros comerciales. Elaboración propia.

Al margen de las cuestiones sociológicas y culturales asociadas al consumo, con carácter general los grandes equipamientos comerciales han ido progresivamente saliendo del núcleo y del municipio central para instalarse en suelos de la primera y segunda corona, todo ello retroalimentando la dinámica de los mercados inmobiliarios en esas zonas, que desde los 80 en adelante fueron nutriendo de nuevos usos residenciales, productivos y terciarios los nuevos desarrollos en los municipios del área.

La interrelación de los criterios de localización y la adecuada articulación de los espacios entre sí pueden convertirse en el hecho diferencial que haga de una ciudad o una parte del área un espacio más cohesionado y equilibrado que otros casos, acorde al modelo de cohesión territorial refrendado en la Agenda Territorial Europea de 2020.

La fuerte adherencia de los grandes centros comerciales a los suelos urbanos no oculta las tensiones que se generan en torno a estos, (especialmente, aunque no solo, en la trama urbana residencial). Los centros no contemplan necesariamente la contigüidad con la trama urbana, localizados en los bordes, la periferia y el territorio metropolitano, se presentan asociados a un viario de alta capacidad. Sin duda, la forma urbana condiciona el planeamiento, y éste a su vez constriñe la posibilidad de nuevos emplazamientos, pero todo indica que -al menos, hasta 2008, agudización de la crisis económica- los centros comerciales se adelantaron a procesos de urbanización o los acompañaban en su desenvolvimiento.

Las cuatro áreas señaladas, Madrid, Barcelona, Valencia y Sevilla, arrojan resultados que permiten contrastar comportamientos. Habida cuenta que una misma estrategia comercial funcionará siempre de un modo diferente en función del escenario en que se ubique y los factores que intervengan (mercado potencial, localización en relación a viario, competencia existente,...) con estos resultados se pone de manifiesto que no solo las pautas sociológicas y económicas en relación al consumo entran en juego, sino que surgen notables diferencias en la distribución de los establecimientos a tenor de condicionantes urbanísticas previas. El grado de "acomodo" entre grandes centros comerciales y sector urbano-metropolitano puede acabar induciendo la atracción de nuevas implantaciones, o la reconducción de nuevos vectores de aperturas. Se insiste en la relación entre grandes superficies a relación inherente de las manchas urbanas (consolidadas y nuevos desarrollos) con las infraestructuras de las comunicaciones, sin descuidar en lo posible el factor cronológico de aparición de los protagonistas. En todo caso, el análisis solo puede avanzar bajo la pauta estratégica de no despreciar los comportamientos que se manejan en materia de movilidad cotidiana y residencial dentro de las áreas, inherentes a las pautas locacionales de los centros de actividad.

Lejos de ser meros contenedores para dispensar productos, estos grandes equipamientos contribuyen a la construcción y modelado de áreas urbanas, tanto con su implantación única o junto a otros desarrollos de suelos terciarios, como a través de los servicios e infraestructuras que demandan. La selección de áreas metropolitanas responde a dos tipos de motivaciones. Se han escogido aquellas que constituyen el rango máximo de configuración supraurbana, con una estructura funcional y una dinámica propia dife- 
renciada del territorio próximo, nivel que en nuestro país alcanzan Madrid y Barcelona. De igual modo resulta relevante contrastar la realidad de éstas con otras áreas de menor proyección territorial que aquéllas pero con dinámicas (procesos de crecimiento, pautas de planificación, modelo de articulación territorial) de complejidad creciente simultaneadas con nuevos desarrollos urbanos que se ven acompañados de estos grandes equipamientos comerciales, como ocurre en Valencia y Sevilla.

Las cuatro áreas presentan un desarrollo metropolitano diferente acorde a la morfología de los núcleos preexistentes y los ejes de crecimiento y conexión interurbanos (y otros elementos no desdeñables, como la topografía o la fachada litoral), sin olvidar los procesos que motivan nuestra delimitación de las áreas metropolitanas españolas (espacios interconectados por los mercados de trabajo, vivienda y servicios). Las diferencias de unas áreas y otras explican en parte las tendencias y pautas de localización de los grandes centros de actividad, centros comerciales entre ellos, y el efecto retroalimentador sobre el planeamiento urbanístico sucesivo.

Los flujos residencia-trabajo en los dos censos, de 2001 y $2011^{7}$ obtenidos a partir de la explotación de las fuentes proporcionadas por el Instituto Nacional de Estadística (INE) ponen de manifiesto la persistencia de Sevilla y Madrid (en mucha menor medida Valencia) como ciudad principal del área respectiva, siendo el catalizador y receptor principal de los desplazamientos por motivo de trabajo (Figura 7). Otra situación paradigmática que persiste entre períodos censales recientes es la proporción claramente superior de los desplazamientos cotidianos por trabajo entre municipios de las coronas del área, tal es el caso de Barcelona.

Figura 7. Movilidad cotidiana por ámbitos de origen-destino en los casos seleccionados. Evolución 2001-2011.

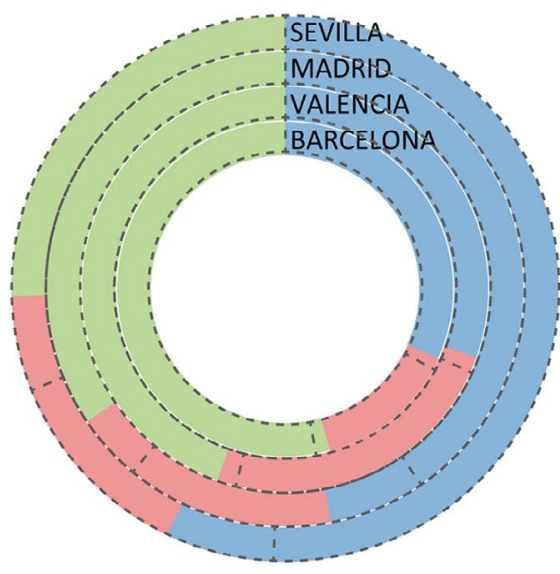

\begin{tabular}{lccc} 
& \multicolumn{2}{c}{ PROPORCIÓN TIPO MOVILIDAD (\%). 2001/2011 } \\
& CORONA-CENTRO & — CENTRO-CORONA & CORONA-CORONA \\
\hline SEVILLA & $57,1 / 50,7$ & $17,5 / 18,2$ & $25,4 / 31,1$ \\
\hline MADRID & $46,9 / 40,4$ & $18,7 / 20,6$ & $34,4 / 39,0$ \\
\hline VALENCIA & $30,5 / 31,8$ & $24,9 / 22,1$ & $44,6 / 46,1$ \\
\hline BARCELONA & $31,8 / 33,0$ & $13,9 / 14,1$ & $54,3 / 52,9$ \\
\hline & & & \\
& & & \\
& &
\end{tabular}

Fuente: INE. Censos de Población de 2001 y 2011. Elaboración propia.

La movilidad cotidiana ilustra el carácter dinámico que queremos resaltar en la conformación de lo metropolitano, en tanto que espacios intermunicipales que participan y se integran en la oferta y la demanda, tanto de servicios y equipamientos como de infraestructuras de los transportes se refiere. Los factores de proyección e instalación de grandes equipamientos, sujetos en todo caso a la demanda y el tipo de servicios que ofrecen, son complejos, diversos y variables de unos casos a otros, pero en cualquier caso, se puede apreciar a continuación el peso alcanzado por los centros comerciales, que no sólo responden a una lógica del mercado de bienes sino -a menudo de modo prevalente- al mercado inmobiliario al que entran los municipios en competencia (Alfonso, 2016). El mayor número de grandes centros comerciales en las áreas de Madrid y Barcelona responde a factores demográficos y a una componente socioeconómica muy intensa como polos de atracción de inversiones y población. El resultado ha sido en ambos casos, aun con notables diferencias entre sí, un desarrollo urbano y metropolitano que no alcanzan el resto de áreas españolas. Veamos algunos matices sobre los comportamientos en cada caso de los seleccionados. Las áreas de Valencia y Sevilla apenas superan entre ambos el número total de centros comerciales localizados en el

7 Desplazamientos medidos y nombrados "centro-corona", "corona-centro", "corona-corona". (para comprender metodología, véase Feria y Vahí, 2010) 
área de Barcelona, y representan prácticamente el 50\% de centros situados en la de Madrid. Esta cifra solo puede ser significativa en tanto que detrás se encuentran operaciones inmobiliarias de alcance variable, pero en nuestro caso el análisis se dirige en otra dirección al conectar los resultados cuantificados con la disposición y la relación de estos establecimientos con el resto de la ciudad y el territorio.

El área de Barcelona se encuentra marcado por un frente litoral variablemente suave y un interior con una orografía muy acentuada en la parte central occidental que marca el desarrollo de los núcleos de población; en ese escenario, los ejes existentes articulan y comunican los nuevos desarrollos urbanos más allá de los pueblos y ciudades históricas.

Figura 8. Grandes centros de actividad y tejido urbano/ejes principales. Área metropolitana de Barcelona

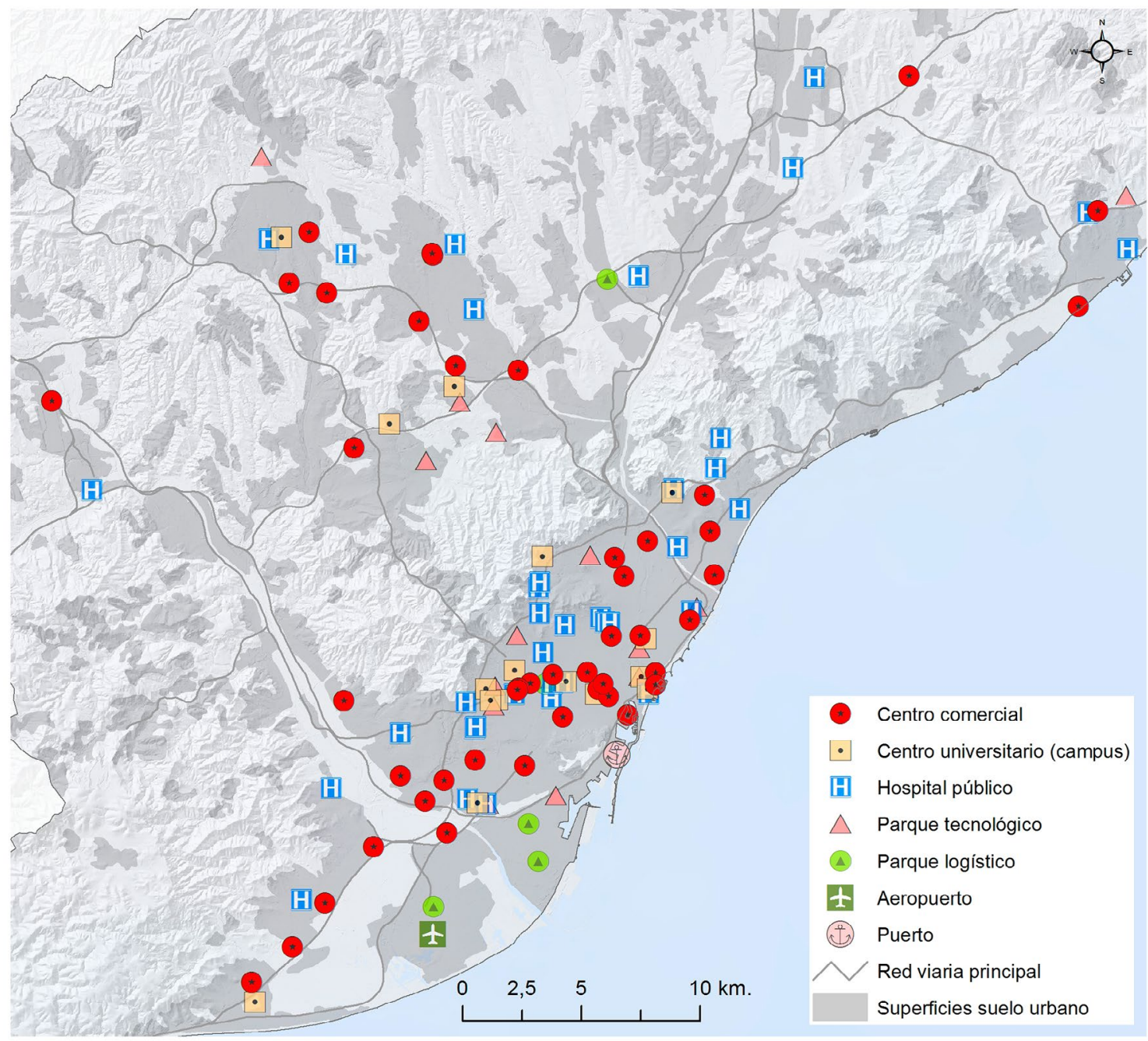

Fuente: Ministerio de Fomento-Instituto Geográfico Nacional (2015, 2016), Ministerio de Sanidad, Servicios sociales e Igualdad (2016), AECC (2012), UNIVERSIA (2015), APTE, Asociación de Centros de Transporte de España (2016). Elaboración propia.

En áreas maduras como ésta se aprecian dinámicas intersectadas y vectores de crecimiento que acaban solapándose, como se aprecia en el cuadrante meridional (Figura 8) sobre el sector de Avenida Diagonal en Barcelona capital, y de ésta en la zona de contacto con los municipios surorientales del Llobregat. Como en el resto de áreas analizadas, la adherencia de los centros comerciales a las ciudades centrales dejan progresivamente paso a localizaciones en las coronas, siendo visible que la presencia responde a emplazamientos sobre viarios de gran capacidad y alta frecuencia de uso (C-31, C-32 y variantes) que se debilita conforme se avanza en sentido centrípeto desde la capital, en consonancia con los datos del mercado de vivienda.

En el caso del área catalana el anclaje de los centros en los bordes urbanos y sobre los principales elementos del viario se observa a la escala del conjunto del área, aunque las condiciones físicas e históricas del 
urbanismo muestran diferentes resultados entre unas zonas y otras del área. Lo más señalado es -como se ha indicado anteriormente- la preferencia de emplazamientos en el sector litoral meridional, que actúa como corredor industrial y de servicios con una potencia mayor que en el extremo opuesto del área. El aeropuerto del Prat y la presencia de determinadas actividades logísticas refuerzan el carácter terciario de la zona como consecuencia, en parte, de un planeamiento de largo recorrido y con una visión integral del espacio como un único ámbito funcional. El reconocimiento de la realidad metropolitana y el principio de cooperación territorial por parte de las administraciones, puede haber incidido en cierto modo sobre la comprensión de una política territorial y un planeamiento urbanístico basado en la identidad metropolitana, al igual que la ágil incorporación perceptiva por parte de la sociedad de un espacio cotidiano ensanchado, para el que se acabó normalizando la redistribución de servicios e infraestructuras de los transportes.

Figura 9. Grandes centros de actividad y tejido urbano/ejes principales. Área metropolitana de Madrid

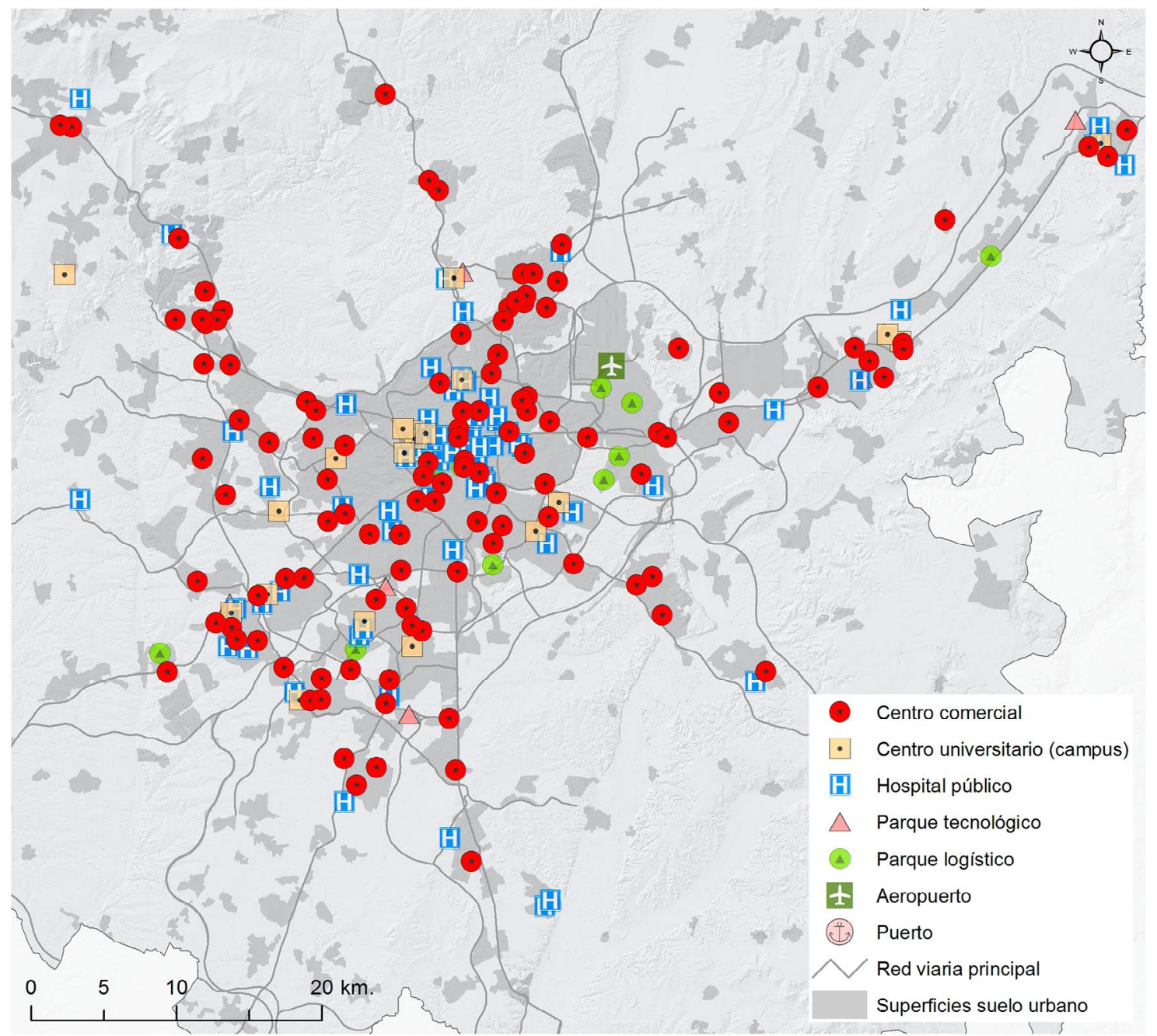

Fuente: Ministerio de Fomento-Instituto Geográfico Nacional (2015, 2016), Ministerio de Sanidad, Servicios sociales e Igualdad (2016), AECC (2012), UNIVERSIA (2015), APTE, Asociación de Centros de Transporte de España (2016). Elaboración propia.

A diferencia de Barcelona, el área de Madrid mantiene el referente de la ciudad central sobre el conjunto. En la Figura 9 se aprecia este rasgo, proyectándose una redistribución de los grandes centros con carácter radiocéntrico de viario de alta capacidad (M-30, M-40, M-50). Ese rasgo ratifica la persistencia de la morfología de la ciudad primacial, que actúa como un foco que se proyecta más allá del núcleo. Esta disposición, que conforma la prolongación de grandes avenidas de la capital, se nutre y complementa con el diseño de trazado radial de ejes, facilitando funciones de accesibilidad y conexiones "centro- corona" y "corona-corona" de la ciudad central con el resto ciudades y territorio metropolitano (AP-6, A-4, A-1, 
A-2). La conurbación en la primera corona del área madrileña se plasma no solo en la ocupación residencial sino también en la intensificación con que aparecen los centros comerciales y de ocio, que salpican la mancha urbana en torno a urbanizaciones y bolsas de nuevos suelos productivos, aunque manteniendo la constante proximidad de un kilómetro o menos a vías de primer orden como la AP-6 (Noroeste).

El policentrismo, con sus particularidades, marcó durante décadas -y continúa a un ritmo diferentela pauta del crecimiento y nuevos desarrollos urbanos que atraen la implantación de grandes centros de actividad. Si bien este hecho se hace patente en los casos anteriores, ha quedado claro que las condiciones preexistentes no constituyen el único criterio determinante en la localización de estos equipamientos. Aun cuando los mercados (vivienda y trabajo) acusaron dinámicas de alcance considerable, otros ejemplos diferentes de las dos grandes áreas acusan ritmos y resultados diversos en lo que al comportamiento de la dinamicidad metropolitana se refiere.

Figura 10. Grandes centros de actividad y tejido urbano/ejes principales. Área metropolitana de Valencia

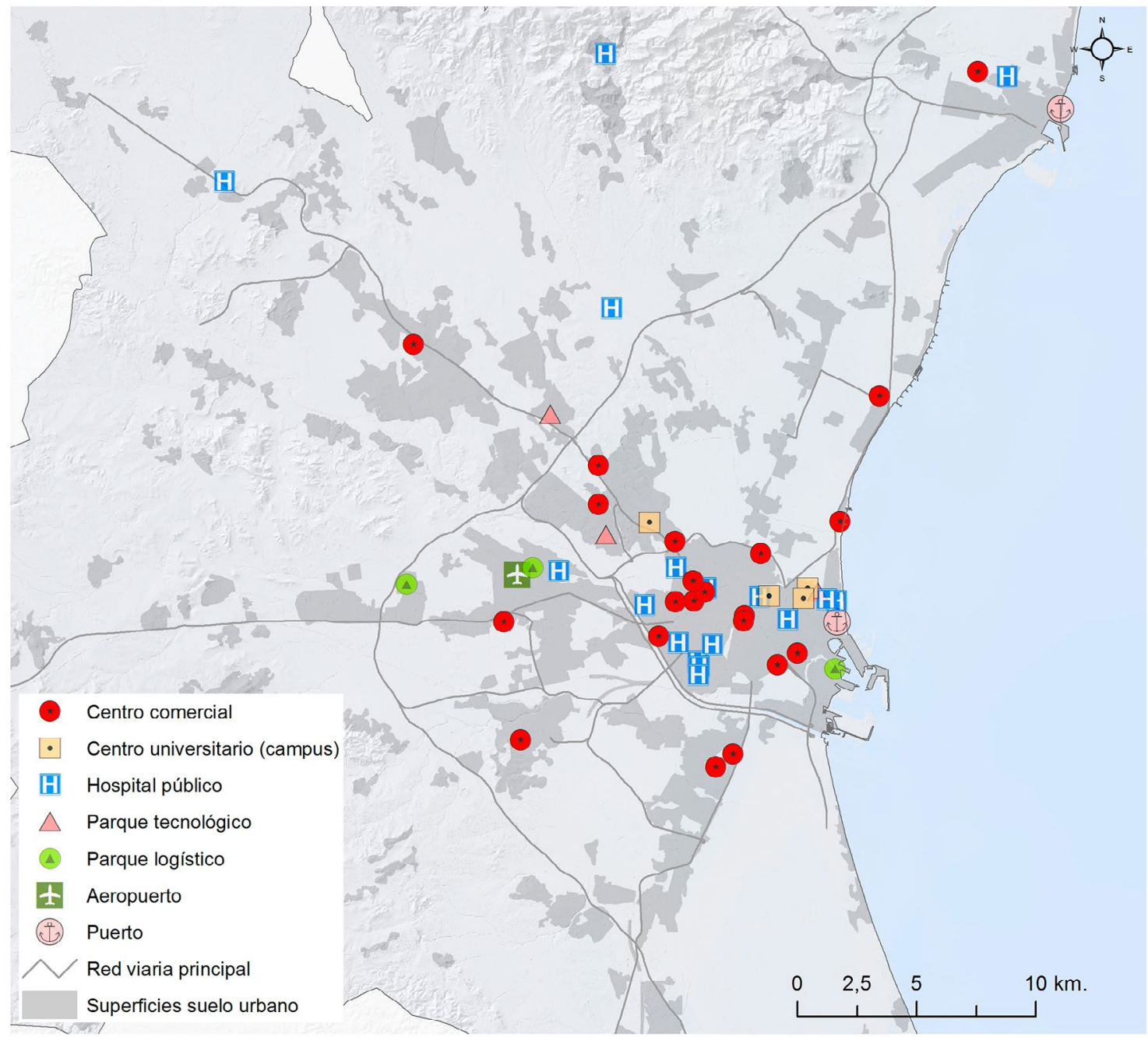

Fuente: Ministerio de Fomento-Instituto Geográfico Nacional (2015, 2016), Ministerio de Sanidad, Servicios sociales e Igualdad (2016), AECC (2012), UNIVERSIA (2015), APTE, Asociación de Centros de Transporte de España (2016). Elaboración propia.

Si Madrid y Barcelona ocupan un primer nivel en el rango de las áreas metropolitanas españolas (población, intensificación de funciones, flujos de movilidad cotidiana,...), Sevilla y Valencia son representativas en un rango diferente, inmediatamente inferior en cuanto a población y complejidad de su proyección. Estas soportan el mayor peso de estos equipamientos dentro del municipio principal. Así ocurre en Valencia, con casi la mitad del cómputo registrado en 2015 (10 de los 22), signo del peso que ejerce la ciudad central aun cuando tras la crisis económica de comienzos de este siglo, se reconocen cam- 
bios sustanciales en la articulación urbana al pasar del modelo radial a diseños de un modelo de mayor complejidad y diversidad (Salom, Albertos y Fajardo, 2015). En este caso, los emplazamientos que saltan de la ciudad central al resto del área se asientan próximos a grandes ejes convencionales: A-7, vinculado a la zona industrial de Almussafes, en el sector meridional, A-3 en la salida hacia Madrid, CV-35, corredor del NO, y desde luego los sectores comprendidos entre algunas de estos ejes y la circunvalación de la autovía del Mediterráneo, AP7, en la zona de Manises, Paterna, Burjasot, hasta el litoral, en Alboraya al norte de Valencia capital. Si bien la movilidad residencia-trabajo acusa los mayores niveles de flujos "corona-corona" de los cuatro casos analizados (Figura 10) ello debe asociarse a la toma de datos en momentos censales específicos (2001 y 2011) mientras que el mapa de grandes superficies comerciales ha ido dibujándose desde los años 80 del pasado siglo, lo que invita a pensar que esa atracción comercial de la capital ha quedado diluida con el paso del tiempo, y es lo que explica la aparición más reciente de algunos grandes establecimientos en municipios de las coronas.

Figura 11. Grandes centros de actividad y tejido urbano/ejes principales. Área metropolitana de Sevilla

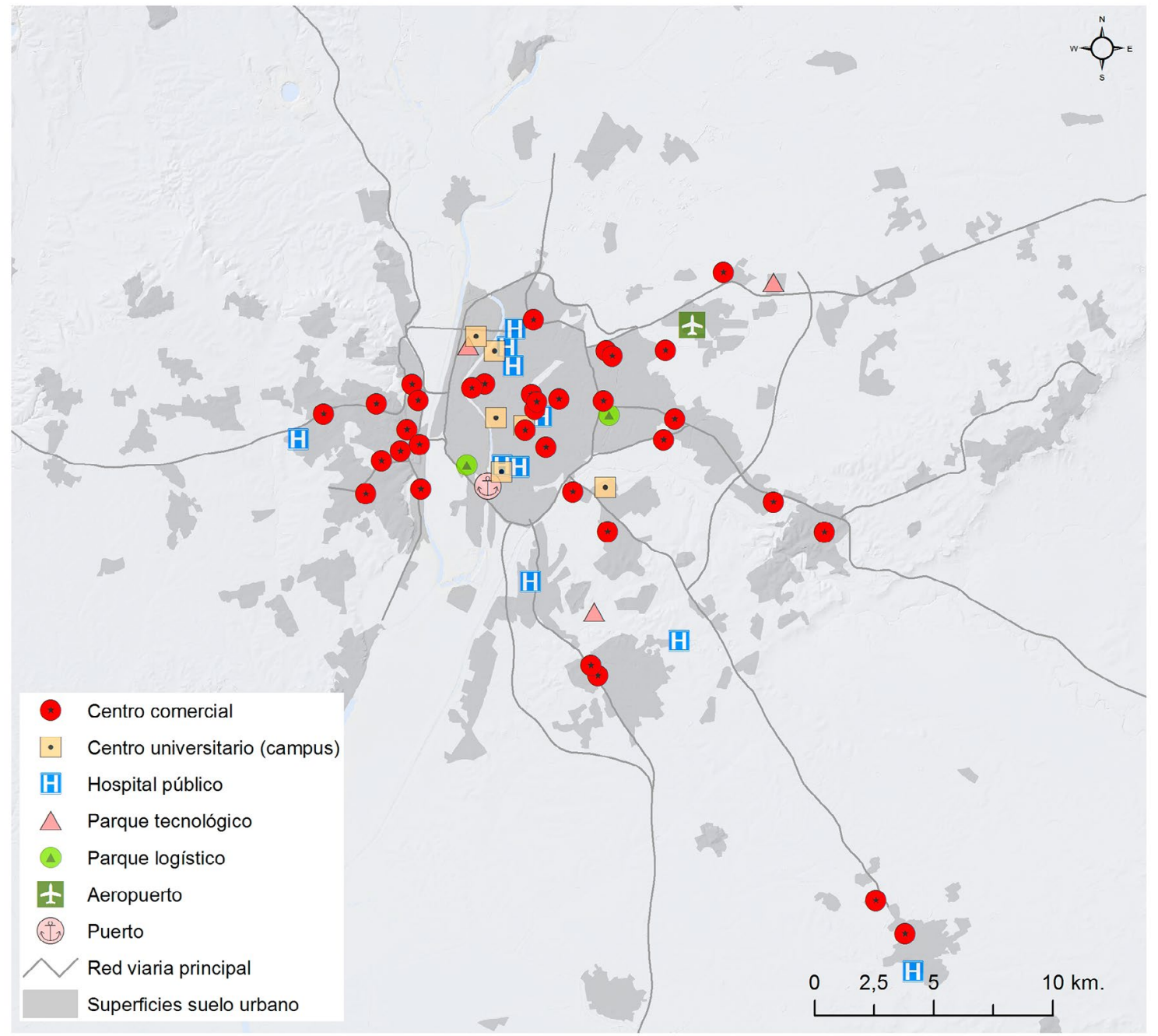

Fuente: Ministerio de Fomento-Instituto Geográfico Nacional (2015, 2016), Ministerio de Sanidad, Servicios sociales e Igualdad (2016), AECC (2012), UNIVERSIA (2015), APTE, Asociación de Centros de Transporte de España (2016). Elaboración propia.

$\mathrm{Al}$ igual que sucede en las restantes áreas, la reconfiguración metropolitana iniciada tras la crisis económica acusada en el mercado inmobiliario desde 2008 ha ido conformando un nuevo diseño del conjunto supramunicipal a raíz de los cambios del mercado y sus repercusiones en la movilidad. Las pautas de apertura y localización del conjunto de los servicios se han visto condicionada por el impasse impuesto por la dinámica de capital y mercado inmobiliario. 
En áreas fuertemente centralizadas, como Sevilla, la localización del gran formato comercial interfiere con un proceso de periferización residencial detectado en municipios de la primera corona, seguido de sucesivos nuevos desarrollos urbanos en sentido centrífugo desde los mismos (Figura 11). Así, la ciudad central mantiene su rol referencial institucional y de servicios al tiempo que asiste a nuevos desarrollos urbanísticos en toda su primera corona, fundamentalmente sobre la meseta del Aljarafe (sector occidental) y hacia el sur y sureste (Dos Hermanas, Alcalá de Guadaíra). También los municipios de la vega del Guadalquivir flanquean sus núcleos históricos de nuevos desarrollos urbanos y ponen en marcha nuevos proyectos al calor de una planificación subregional que instaura las "áreas de oportunidad". Con o sin el amparo de estas figuras, los centros de actividad han ido surgiendo al calor de los nuevos crecimientos, y acaba reproduciéndose una vez más la condición de proximidad con el viario de alta capacidad.

A la vista de la figura 11 resalta la localización de los centros fuera de la capital, sobre la zona del Aljarafe, próximos siempre a la SE-30 y/o a la A-49, que se convierten (por intensidad de flujos) en los ejes que articulan y distribuyen los desplazamientos más numerosos. Junto a la A-49, Autopista del V Centenario, principal corredor funcional sobre la corona, la A-92, A-4 y la A-376 conforman los principales ejes radiales que convergen en el núcleo del área sevillana. Aun existiendo dificultades de carácter económico en el mercado inmobiliario, Sevilla y su área conocerán en breve espacio de tiempo algunas aperturas más, anunciadas sobre los principales ejes de comunicación como la SE-30 (próximo al Puerto de Sevilla) que denotan una perspectiva continuista de implantación de grandes equipamientos comerciales. A qué ritmo se produzca, como ocurre y ocurrirán en las demás áreas analizadas, y en el resto de España, es un hecho aún por conocer y analizar, pero la información vertida apunta a que estas fórmulas comerciales se mantienen en buena forma y continúan dispuestas a su expansión. Aun cuando las grandes enseñas apuestan por diversificar sus estrategias de localización, tamaño, oferta,... todo apunta a que seguirán abriéndose grandes superficies comerciales en tanto el mercado inmobiliario brinde estas posibilidades a los inversores y comercializadores.

\section{Discusión de resultados}

Del análisis realizado se desprende que las cuatro áreas se ven envueltas en modelos de crecimiento distintos en los que servicios e infraestructuras están abocados a protagonizar procesos urbanísticos de diferente calado. Las características sociodemográficas y económicas de esos espacios se ven en ocasiones sobrepasadas por operaciones que vienen de la mano de los mercados de suelos y capital inversor. Estos han dinamizado -con extraordinaria potencia en ocasiones- nuevos desarrollos urbanos, aunque bien es cierto que tanto en ellas como en el conjunto del país se ha acusado fuertemente la crisis y las pérdidas de capital (consecuencia del paréntesis financiero internacional, muy acusado entre 2006 y 2015). Con todo, la decisión de considerar la fase más reciente de estudio, con cabida para una coyuntura crítica extraordinaria no deriva en un diagnóstico sesgado que hiciera dudar de la certeza en las conclusiones. En ese sentido, las otras fases contempladas contuvieron también momentos críticos de uno u otro calado con las consiguientes repercusiones en el mercado inmobiliario e inversor.

La orientación de los mercados, en todo caso, se afianzó sobre el sector inversor inmobiliario y actualmente reemprende la andadura interrumpida hacia 2008, comprometiendo entornos urbanos y la calidad ambiental. A fuerza de continuar con un planeamiento orientado a dotar de sistemas para nuevos usos residenciales, productivos, terciarios, se hace urgente una llamada a la prudencia para trabajar en la reconducción de esta trayectoria.

La agilización de los desplazamientos motivados por la afluencia a grandes equipamientos comerciales se apoya en el tándem que conforman los espacios de centralidad (de uno tipo u otro) y la calidad del transporte, medida no sólo por la presencia de las infraestructuras sino de manera paulatinamente creciente por la existencia de una oferta en materia de transporte público y una apuesta por la intermodalidad que no termina de madurar aún. Usuarios individuales e instancias públicas y responsables de la gestión tendrán que asumir la necesidad de cambio (a expensas de otro cambio que se precipita y resulta irreversible, el cambio climático) y adaptarse a la contención del gasto energético que se deriva del abuso del vehículo particular.

En cuanto al análisis crítico de las preferencias a la hora de implantar un CC, el siguiente gráfico sintetiza a las claras lo que ya se ha identificado en los casos de estudio. Existe una tendencia generalizada a establecerse en espacios más alejados al núcleo central (con matices y tempos distintos, como indica el freno reciente de esta tendencia en las dos áreas más grandes, incluso se revierte en la de Barcelona) (Figu- 
ra 12). Y de nuevo se vuelve al peso adquirido progresivamente por las infraestructuras en relación a estas localizaciones. Los casos analizados denotan uniformidad al respecto y se ve cómo los CC acortan sus distancias (en promedio) a la carretera más próxima, obteniéndose un resultado inverso en el dato acumulado del resto de áreas en su conjunto, que habría de ser objeto de un análisis diferente al que aquí se presenta.

Figura 12. Evolución en la localización de Centros Comerciales en relación a tejido urbano y ejes principales (distancia promedio).
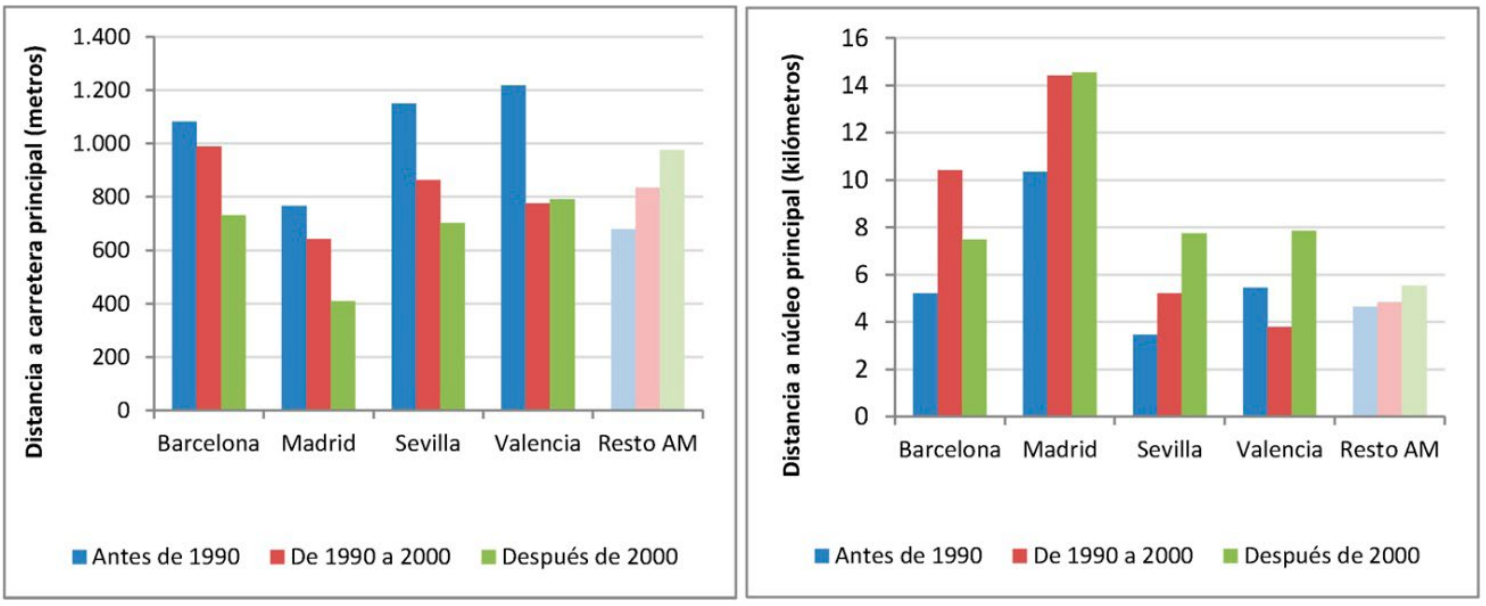

Fuente: CLC (Ministerio de Fomento-Instituto Geográfico Nacional (2016) y BTN100 Ministerio de Fomento-Instituto Geográfico Nacional (2015). AECC, 2012 y webs propias de enseñas centros comerciales. Elaboración propia.

En este punto de la discusión encontramos cuestiones subsumidas a lo largo del trabajo, claves para la mejora de la articulación urbana y metropolitana, tratadas por otros investigadores de modo expreso y con más profundidad, pero que es necesario aludir. La implantación de los CC hasta el momento ha seguido la pauta locacional previsible en todas las actividades económicas, productivas y terciarias. Pese a la incorporación de nuevos medios para la venta y los servicios en general, vía electrónica, las ventajas a veces concedidas a comercializadoras/promotoras pueden a corto plazo dar respuesta a los intereses de estas entidades, pero dar al traste con la agilización de la accesibilidad en función de los flujos cotidianos, los modos de transporte o la relación con sectores urbanos/interurbanos relativamente remotos o distantes. La no interiorización de las tensiones generadas por parte de los responsables de los CC y la ausencia de criterios firmes al respecto desde las administraciones implicadas pueden condicionar el plan para otros suelos, otros usos y usuarios.

La urgencia por abordar firmemente la cuestión de la accesibilidad no oculta lo relevante que el modo para abordarla resulta para el gobierno de las ciudades y ámbitos metropolitanos. En el marco de las políticas actuales, la llamada a los procesos de participación está presente a cualquier nivel y escala de la que se trate. La puesta en marcha de una gobernanza asociada a las movilidad y la modalidad se hace necesaria si se estima que la prioridad recae en el modelo que se desea construir, y no en medidas cambiantes asentadas en los programas electorales de los gobiernos municipales y supralocales. Autores como Savy (2015) o Banister (2011) grandes conocedores en materia de transportes insisten en la adecuación de los escenarios urbanos a los parámetros intensos y cambiantes de los flujos y modos de transportes. En España, herramientas como el PEIT pueden contribuir a reconducir esta situación como en realidad lo hacen (aunque visiblemente tensionado por la dinámica inversora inmobiliaria). Las investigaciones sobre las herramientas de modelización avanzan, se aprecia la voluntad creciente de integrar las soluciones desde todos los escenarios y protagonistas. Consideramos que los centros comerciales desempeñan en la actualidad un papel importante en la configuración y articulación de nuestras ciudades, del mismo modo que confiamos en la oportunidad de asumir protagonismo como pilotos de una reconducción del modelo.

\section{Conclusiones}

La habitabilidad y la calidad de vida en los distintos sectores o zonas de las áreas dependen de la disponibilidad de los servicios y del grado de accesibilidad, siendo ésta un requisito sine qua non de lo- 
calización de este tipo de equipamientos, que define su posición y ámbito de influencia espacial respecto a la población a la que está destinada; en ese contexto se tendrían que asegurar las conexiones en un umbral de condiciones socioeconómicas y ambientales satisfactorio. La satisfacción vendrá dada por la capacidad de respuesta a la mejora de las condiciones de los desplazamientos en relación tiempo y distancia, y alternativas de modalidad del transporte. Si bien la orientación sobre las políticas de movilidad metropolitana viene marcando desde hace años en España una serie de medidas para alcanzar mínimamente dichas garantías, las tensiones sostenidas por dichas políticas proceden de las contradicciones con las que se topan en materia de planeamiento urbanístico y la debilidad que aún arrastra el concepto de intermodalidad en buena parte de nuestro país por motivos diversos como la insuficiencia inversora en transporte público y la limitada concienciación social respecto a la oferta existente. La intensificación de la movilidad motorizada y uso del vehículo privado han participado, y aún perduran, en la conformación del fenómeno metropolitano, aun cuando el planeamiento urbanístico sigue contenido en los límites municipales, obviando cuestiones de alcance supralocal.

Si bien los criterios locacionales de las actividades económicas son inherentes al trazado que garantiza la afluencia de usuarios (personas, mercancías, información,... vehículos) el emplazamiento de los grandes establecimientos y complejos requiere en esta nueva ciudad metropolitana una planificación exhaustiva e integral que regule este y otros aspectos como la saturación de bolsas de suelos (sobre las periferias urbanas, en las coronas metropolitanas), auténticos contenedores de equipamientos hoy, sin consideraciones ambientales a medio y largo plazo. Eficiencia energética y equidad (socialmente al alcance de todos los sectores sociales) habrán de introducirse con mayor fuerza que la producida hasta el momento en nuestro país, tanto por parte de las políticas de movilidad como por parte de la sociedad misma. Queda fuera de toda duda que la afluencia de los compradores a los centros comerciales hoy día está sujeta a parámetros como la diversidad de la oferta, accesibilidad y grado de innovación, y/o la combinación de -si no todas- al menos algunas de estas motivaciones (Beavon, 1981). En definitiva, la elección del establecimiento por el usuario está supeditada a la adecuación del emplazamiento a las necesidades del propio centro, y a las relaciones que éste entabla en un amplio alcance, dentro del área (y a veces sobrepasándolo) con el resto del lugar elegido. Si además existe una presencia reguladora de cierto alcance a través de la planificación territorial y el planeamiento urbanístico se impone la observación sobre la idoneidad del modelo territorial, y la adecuada localización de los grandes centros comerciales como elementos articuladores del territorio, que contribuyen (o al menos pueden aspirar a ello) a armonizar la función urbana. Algunos pasos dados desde instancias diversas pueden ser un punto y seguido sobre la propuesta que aquí se plantea y en ese sentido cabe explorar resultados emanados de la Ley de Comercio Interior de Andalucía (Junta de Andalucía, 2014), en la que se aborda la integración de la ordenación comercial y el planeamiento urbano.

Por último, los nuevos procesos urbanizadores también se reconocen en los aportes de la geografía de la accesibilidad (Gutiérrez y Mata, 2010; Paris, 2013) y es necesario profundizar en el desarrollo de garantías de esta condición funcional indispensable por parte de los grandes complejos de comercio y ocio; urge evaluar el alcance de su incorporación por parte de los centros comerciales y reconsiderar el balance sobre la movilidad cotidiana en el entorno de los establecimientos y en el conjunto urbano y metropolitano. En definitiva, tras décadas de existencia y estrategias diversas, cabe esperar una adecuación a los nuevos escenarios, en los que el cambio climático desempeña un papel primordial. De ahí la propuesta de romper inercias pretéritas y asumir tareas para con un planeamiento urbanístico responsable y coherente. Al mismo tiempo, la movilidad urbana habrá de precipitarse a cambios y nuevos hábitos de modalidad, fuentes de energía, organización temporal de las actividades, etc. a fin de contribuir con un modelo de ciudad sostenible, demasiado tiempo postergado.

\section{Agradecimientos}

Expresamente al equipo de investigadores del Proyecto "Dinámicas y transformaciones territoriales, funcionales y sociales de las áreas metropolitanas españolas en un horizonte de sostenibilidad", financiado en el marco del Plan Estatal de Investigación Científica y Técnica y de Innovación 2013-2016, cód. CSO2014-55780-C3-1-P. De modo especial, nuestro agradecimiento al Dr. Feria Toribio por las recomendaciones y reflexiones compartidas en torno al tema de estudio así como la orientación en la difusión de los resultados. 


\section{Referencias}

Alfonso, F. (2016). El potencial dinamizador de los grandes equipamientos comerciales en espacios metropolitanos. Un análisis comparado en tres realidades españolas. DOCFRADIS, Cátedra Fundación Ramón Areces de Distribución Comercial, Universidad de Oviedo. № 1605. Recuperado de http:// econpapers.repec.org/paper/ovrdocfra/1605.htm

Arroyo, M. (2001). La contraurbanización: un debate metodológico y conceptual sobre la dinámica de las áreas metropolitanas. Scripta Nova. Revista electrónica de Geografía y Ciencias Sociales, vol. V, núm. 97. Recuperado de http://www.ub.edu/geocrit/sn-97.htm

Asociación de Centros de Transporte de España (2016). Mapa de Centros asociados. Recuperado de http://www.acte.es/pagEstatica.aspx?id=7

Asociación Española de Centros Comerciales (AECC) (2015). Historia de un éxito. Recuperado de http://www.aedecc.com/uploads/secciones/galeria_documentos/Cuadro_Historia_de_un_xito_ Mayo_2015.pdf

Asociación de Parques Científicos y Tecnológicos de España (APTE) (2015). Relación de los miembros de APTE. Recuperado de http://www.apte.org/es/parques-miembros.cfm

Banister, D. (2011). The trilogy of distance, speed and time. Journal of Transport Geography, 19, 950-959. https://doi.org/10.1016/j.jtrangeo.2010.12.004

Beavon, K. (1981). Geografía de las actividades terciarias. Una reinterpretación de los lugares centrales. Barcelona: Oikos Tau.

Berry, B. J. L., Goheen, P. \& Goldstein, H. (1970). Problems and perspectives of defining the metropoli, in Berry, B \& Horton, F. Geographic Perspectives on Urban Systems. Englewood Cliffs: Prentice Hall, 250-276.

Burriel de Orueta, E.L. (2008). La "década prodigiosa" del urbanismo español (1997-2006). Scripta Nova. Revista Electrónica de Geografía y Ciencias Sociales, vol. XII, núm. 270 (64). Recuperado de http:// www.ub.es/geocrit/sn/sn-270/sn-270/sn-270-64.htm

Dematteis, G. (1998). Suburbanización y periurbanización. Ciudades anglosajonas y ciudades latinas. En FJ. Monclús (ed.), La ciudad dispersa. Suburbanización y nuevas periferias. Barcelona: CCCB.

Dematteis, G. (2004). En la encrucijada de la territorialidad urbana. En A. Martin Ramos (coord.), Lo urbano en 20 autores contemporáneos. Barcelona: Ediciones UPC.

Feria Toribio, J.M. (2004). Problemas de definición de las áreas metropolitanas en España. Boletín de la Asociación de Geógrafos Españoles, 38, 85-99. Recuperado de https://dialnet.unirioja.es/servlet/ articulo? codigo $=1079120$

Feria Toribio, J.M. (2008). Un ensayo metodológico de definición de las áreas metropolitanas en España a partir de la variable residencia-trabajo. Investigaciones Geográficas, 46, 49-68. https://doi.org/10.14198/ INGEO2008.46.03

Feria, J.M. y Vahí, A. (2010). Distribución modal de la movilidad cotidiana metropolitana. En J.M. Feria y J.M. Albertos (eds.), La ciudad metropolitana en España: procesos urbanos en los inicios del siglo XXI. Cizu Menor (Navarra): Civitas-Thomson Reuters.

Fundación UNIVERSIA (2015). Portal de Universidades. Universidades de España. Recuperado de http:// www.universia.es/universidades

García Palomares, J.C. y Gutiérrez Puebla, J. (2007). La ciudad dispersa: cambios recientes en los espacios residenciales de la Comunidad de Madrid. Anales de Geografía de la Universidad Complutense, 27(1), 4567. Recuperado de http://revistas.ucm.es/index.php/AGUC/article/view/AGUC0707110045A/30912

Gutiérrez, J. y Mata, R. (2010). Tecnologías de la Información Geográfica para el buen gobierno de la ciudad y el territorio. Ciudad y territorio: Estudios territoriales, No 165-166, 421-430.

Guy, C. (2006). Planning for Retail Development: A Critical View of the British Experience. London-New York: Routledge.

Hall, P. (2004). Megaciudades, ciudades mundiales y ciudades globales. En A. Martin Ramos (coord.), Lo urbano en 20 autores contemporáneos. Barcelona: Ediciones UPC. 
Instituto Nacional de Estadística (INE)(2016). Censos de población y viviendas 2001 y 2011. Recuperado de http://www.ine.es/censos2011_datos/cen11_datos_inicio.htm

Junta de Andalucía. Decreto-Ley 12/2014, de 7 de octubre, por el que se modifica el Decreto Legislativo 1/2012, de 20 de marzo, por el que se aprueba el texto refundido de la Ley del Comercio Interior de Andalucía. BOJA núm. 202 de 16 de Octubre de 2014.

Ministerio de Fomento-Instituto Geográfico Nacional (2015). Base Cartográfica Nacional 1:100.000 (BTN100). Recuperado de https://www.ign.es/ign/layoutIn/actividadesBDGbtn100.do

Ministerio de Fomento-Instituto Geográfico Nacional (2016). Proyecto CORINE Land Cover - Instituto Geográfico Nacional. Recuperado de https://www.ign.es/ign/layoutIn/corineLandCover.do

Ministerio de Sanidad, Servicios sociales e Igualdad (MSSI) (2016). Catálogo Nacional de Hospitales. Recuperado de http://www.msssi.gob.es/ciudadanos/prestaciones/centrosServiciosSNS/hospitales/ docs/CNH2016.pdf

Observatorio de la Movilidad Metropolitana (OMM) (2016). Informe de la Movilidad Metropolitana, 2014. Recuperado de http://www.observatoriomovilidad.es/es/publicaciones/informes.html

Paris, M. (2013). De los centros urbanos consolidados a los lugares de centralidad: una propuesta metodológica para su estudio. Ciudades, 16 (1) 47-69. Recuperado de http://uvadoc.uva.es/ bitstream/10324/10345/1/CIUDADES-2013-16-DELOSCENTROS.pdf

Salom, J. y Albertos, J.M. (2014). Delimitación y caracterización de los nuevos espacios urbanos valencianos. Boletín de la Asociación de Geógrafos Españoles, (64), 127-149. Recuperado de https:// dialnet.unirioja.es/servlet/articulo? codigo $=4653662$

Salom, J., Albertos, J.M. y Fajardo, F. (2015). Crisis y reestructuración en el área metropolitana de Valencia. Revista de Estudios Andaluces, 32, 1-21. Recuperado de http://institucional.us.es/revistas/ andaluces/32/es/A_JULIA\%20SALOM\%20 2015.pdf

Savy, M. (2015). Nouveaux lieux, nouveaux flux. Les mobilités de l'avenir. París: Odile Jacob.

Serrano, J.M. (2001). Accesibilidad territorial en España. Autopistas y autovías. Papeles de geografía, 33, 133-158.

Serrano, J.M. (2006). El plan estratégico de infraestructuras y transportes (2005-2020), sus planteamientos frente a la mejora de la vertebración territorial española. Nimbus: Revista de climatología, meteorología y paisaje, (17-18) 177-204.

Tamames, R. (2009). Para salir de la crisis global: Análisis y soluciones. Propuestas para España y Latinoamérica. EDAF, Madrid.

Vahí, A. y Feria, J.M. (2007). Estructuras urbanas y grandes formatos comerciales: el ejemplo de las áreas metropolitanas andaluzas, en Ería: Revista cuatrimestral de geografía, 72, 35-54. Recuperado de http:// www.unioviedo.es/reunido/index.php/RCG/article/view/1569

Vahí, A., Rodríguez, V. y Hurtado, C. (2015). Functional centrality and effects on metropolitan reconfiguration, en Boletín de la Asociación de Geógrafos Españoles, 68, 451-454. Recuperado de https://dialnet.unirioja.es/servlet/articulo?codigo $=5137042$

Williams, K., Jenks, M. \& Burton, E. (eds) (2001). Achieving Sustainable Urban Form. London: Spon Press, Group Taylor \& Francis. https://doi.org/10.1016/S0264-8377(01)00010-2 